\title{
Time-Delayed Interactions in Networks of Self-Adapting Hopf Oscillators
}

\author{
Julio Rodriguez, ${ }^{1}$ Max-Olivier Hongler, ${ }^{2}$ and Philippe Blanchard ${ }^{1}$ \\ ${ }^{1}$ Fakultät für Physik, Universität Bielefeld, 33501 Bielefeld, Germany \\ ${ }^{2}$ STI/IMT/LPM, Ecole Polytechnique Fédérale de Lausanne, 1015 Lausanne, Switzerland
}

Correspondence should be addressed to Max-Olivier Hongler; max.hongler@epfl.ch

Received 30 August 2012; Accepted 24 September 2012

Academic Editors: A. Carpio, J. V. Stokman, and C. Zhu

Copyright (c) 2013 Julio Rodriguez et al. This is an open access article distributed under the Creative Commons Attribution License, which permits unrestricted use, distribution, and reproduction in any medium, provided the original work is properly cited.

\begin{abstract}
A network of coupled limit cycle oscillators with delayed interactions is considered. The parameters characterizing the oscillator's frequency and limit cycle are allowed to self-adapt. Adaptation is due to time-delayed state variables that mutually interact via a network. The self-adaptive mechanisms ultimately drive all coupled oscillators to a consensual cyclostationary state, where the values of the parameters are identical for all local systems. They are analytically expressible. The interplay between the spectral properties of the coupling matrix and the time delays determines the conditions for which convergence towards a consensual state takes place. Once reached, this consensual state subsists even if interactions are removed. In our class of models, the consensual values of the parameters depend neither on the delays nor on the network's topologies.
\end{abstract}

The farther back you can look, the farther forward you are likely to see Winston Churchill

\section{Introduction}

The harmonic excitation of an elementary-damped harmonic oscillator

$$
\underbrace{\ddot{x}(t)+\mathrm{a} \dot{x}(t)+\mathrm{f} x(t)}_{\text {system }}=\underbrace{\mathrm{r} \sin (\mathrm{w} t)}_{\text {environment }}
$$

with $a, f, r$ and $w>0$ produces the well-known asymptotic response (c.f. [1]):

$$
x(t)=\mathrm{k} \cos (\mathrm{w} t-\vartheta)
$$

where $k$ and $\vartheta$ depend on the control parameters a, $r$, and $\mathrm{w}$. By construction, the oscillating environment here materialized by the input $r \sin (w t)$ is totally insensitive to the oscillator $x(t)$, implying that $x(t)$ in (1) is slaved by the external forcing. The next stage of complexity is to replace the harmonic oscillator in (1) by a Lienard system:

$$
\ddot{x}(t)+\mathrm{R}(x(t), \dot{x}(t))+\mathrm{f} x(t)=\mathrm{r} \sin (\mathrm{w} t),
$$

where $\mathrm{R}(x(t), \dot{x}(t))$ is a nonlinear controller. In absence of external excitation in (3) (i.e., when $r=0$ ), we assume $R$ to asymptotically drive the orbits towards a stable limit cycle which is independent of the initial conditions - the paradigmatic illustration being here the Van der Pol oscillator. When $r \neq 0$ and for a suitably selected range of parameters, the time asymptotic response of (3) can be qualitatively written as (c.f. $[2,3])$

$$
x(t)=\mathrm{S}(t)
$$

with $\mathrm{S}(t)$ being a a synchronized signal with the same periodicity as the environment (i.e., $\mathrm{S}(t+(2 \pi / \mathrm{w}))=\mathrm{S}(t))$. By construction, the external forcing $r \sin (w t)$ in (3) is, as before, insensitive to the Lienard oscillator. In the resulting synchronized regime, the oscillator $x(t)$ is caught by the external excitation-in other words, the system adjusts itself to the environment but the environment remains insensible to the system. Observe that the dynamical response given by (4) only subsists as long as $r \sin (w t)$ acts on the system. That is, as soon as the environment effect is removed (i.e., $r=0$ in (3)), 
the system (i.e., the limit cycle oscillator), after a transient time, recovers its original behavior-converges towards its limit cycle.

In our present paper, we will extend the previous classical system-environment relationship in order to allow more realistic situations where mutual interactions permanently affect both the system and the environment. Such adaptive mechanisms can modify individual dynamics on a permanent basis. To stylize this new situation, the basic dynamics given by (3) is modified as

$$
\begin{aligned}
& \ddot{x}(t)+\mathrm{R}(x(t), \dot{x}(t))+f(t) x(t)=\mathrm{r} \sin (\omega(t) t), \\
& \dot{f}(t)=\mathrm{A}^{f}(x(t), \dot{x}(t)), \quad \dot{\omega}(t)=\mathrm{A}^{\mathrm{w}}(x(t), \dot{x}(t)),
\end{aligned}
$$

where $f \mapsto f(t)$ and $\mathrm{w} \mapsto \omega(t)$ are no longer constant parameters but variables of the global dynamics (and hence time dependent) and the functions $A^{f}$ and $A^{w}$ capture the mutual adaptation of the system-environment dipole. Hence, the system of (5) has now to be considered globally-the system and its environment, are allowed to adaptively coevolve.

The particular case of (5) when $A^{f} \equiv 0$ (i.e., $f(t):=f$ for all $t$ ) has been studied in $[4,5]$. This type of dynamical system provides a cornerstone of bioinspired robotics where legs (or arms) of robots may be modeled by damped oscillators as (1) (i.e., $\mathrm{R}(x(t), \dot{x}(t))=\mathrm{a} \dot{x}(t))$. To ensure a maximum leg stride, the damped oscillators must be excited by $r \sin (\omega(t) t)$ at the damped oscillator's resonant frequency (i.e., $\omega(t) \simeq \sqrt{f}$ for all $t$ ). However, due to structural changes on the robot (adding load, lengthening legs), the resonant frequency of the damped oscillator has to be adjusted: $f \mapsto \bar{f}$. Hence, $A^{w}$ drives $\omega(t)$ towards the value $\bar{f}$ to systematically guarantee the excitation at resonant frequency and thus maximum leg stride. Frequency adaptation (as well as amplitude adaption) is also important in movement assistance (e.g., retrain the nervous system, assist people with movement disorder), where robots and human beings must work in synchronous. An example of an exoskeleton for the human elbow was studied in $[6]$.

The case where neither $A^{f}$ nor $A^{w}$ is trivial has been covered in $[7,8]$, where the authors not only considered two adaptive coupled limit cycle oscillators, but $n$ mutually interacting through a network. Here, self-adapting oscillators can be applied to robot formation modeling. Each individual robot belonging to a swarm, circulating around a specific point, adapts its angular velocity in order to lower the amount of exchanged information to maintain the formation. Selfadaptation in networks is also considered in [9], where here the control signals (and not the local systems) of the variables of the CPG adapt, and this, to quickly react to new situations and produce several different behavioral patterns.

Building on what has been done in previous contributions $[4,5,7,8]$, we here consider a network of limit cycle oscillators interaction with time-delayed state variables. The general form of our dynamical system in the phase-radius coordinates (i.e., polar coordinates $\phi_{k}$ and $r_{k}$ ) is

$$
\begin{aligned}
\dot{\phi}_{k}(t)= & \mathrm{P}\left(\phi_{k}(t), r_{k}(t) ; \Omega_{k}\right) \\
& -\mathrm{c}_{k} \frac{\partial \mathrm{V}}{\partial \phi_{k}}(\phi(t-\mathrm{t}), r(t-\mathrm{t})), \quad k=1, \ldots, n,
\end{aligned}
$$

$$
\begin{aligned}
\dot{r}_{k}(t)= & \underbrace{\mathrm{R}\left(\phi_{k}(t), r_{k}(t) ; \Omega_{k}\right)}_{\text {local dynamics }} \\
& -\underbrace{\mathrm{c}_{k} \frac{\partial \mathrm{V}}{\partial r_{k}}(\phi(t-\mathrm{t}), r(t-\mathrm{t}))}_{\text {coupling dynamics }}, \quad k=1, \ldots, n,
\end{aligned}
$$

where $\mathrm{P}$ and $\mathrm{R}$ govern the local dynamics, $\phi=\left(\phi_{1}, \ldots, \phi_{n}\right)$ and $r=\left(r_{1}, \ldots, r_{n}\right)$ are the state variables, $\Omega_{k}$ is a parameter set determining the local characteristics and $\mathrm{c}_{k}>0$ are coupling strengths. The coupling dynamics is the gradient of a potential $\mathrm{V}$ depending on state variables with time delay $\mathrm{t}$. Adaptation of the local dynamics is accomplished by letting the constant parameters $\Omega_{k}$ become time-dependent (i.e., $\left.\Omega_{k} \mapsto \Omega_{k}(t)\right)$ with their own dynamics given by adaptive mechanisms $\mathrm{A}_{k}$. Delays being ubiquitous in applications, their influence on adaptive processes is worth to be investigated, and so the general formalization is

$$
\begin{aligned}
\dot{\phi}_{k}(t)= & \mathrm{P}\left(\phi_{k}(t), r_{k}(t), \Omega_{k}(t)\right) \\
& -\mathrm{c}_{k} \frac{\partial \mathrm{V}}{\partial \phi_{k}}(\phi(t-\mathrm{t}), r(t-\mathrm{t})), \quad k=1, \ldots, n \\
\dot{r}_{k}(t)= & \underbrace{\mathrm{R}\left(\phi_{k}(t), r_{k}(t), \Omega_{k}(t)\right)}_{\text {local dynamics }} \\
& -\underbrace{\mathrm{c}_{k} \frac{\partial \mathrm{V}}{\partial r_{k}}(\phi(t-\mathrm{t}), r(t-\mathrm{t}))}_{\text {coupling dynamics }}, \quad k=1, \ldots, n \\
\dot{\Omega}_{k}(t)= & \underbrace{\mathrm{A}_{k}(\phi(t-\mathrm{t}), r(t-\mathrm{t})), \quad k=1, \ldots, n .}_{\text {adaptive mechanism }}
\end{aligned}
$$

We therefore confer to $\Omega_{k}$ the status of variables of the whole dynamical system. Let us remark that in this present contribution, adaptation occurs in the local systems. Note that in [10], the authors introduce, with the help of the speedgradient method, an adaptive mechanism on the coupling constant that multiplies the delayed interactions.

This paper is organized as follows: in Section 2 we define the three components that together form the global system. We then discuss the dynamics of our model in Section 3. An application is presented in Section 4 which is then followed by some numerical experiments in Section 5. Finally, we conclude in Section 6.

\section{Networks of Hopf Oscillators with Adaptive Mechanisms}

We now present explicitly the local and coupling dynamics as well as the adaptive mechanisms on which we will focus.

2.1. Local Dynamics. Each node of the network is equipped with a local dynamical system. In this contribution, a local 
system is a Hopf oscillator presented here in its polar coordinates:

$$
\begin{aligned}
& \mathrm{P}\left(\phi_{k}, r_{k} ; \Omega_{k}\right)=\mathrm{w}_{k}, \quad k=1, \ldots, n, \\
& \mathrm{R}\left(\phi_{k}, r_{k} ; \Omega_{k}\right)=-\left(r_{k}^{2}-\mathrm{r}_{k}\right) r_{k}, \quad k=1, \ldots, n .
\end{aligned}
$$

The state variables are $\left(\phi_{k}, r_{k}\right)$ and $\Omega_{k}=\left\{\omega_{k}, r_{k}\right\}$ are, for the time being, fixed and constant parameters. The parameter $\mathrm{w}_{k}$ controls the frequency of the $k$ th oscillator given by the phase dynamics $P$. The radial dynamics $R$ produces a stable circular limit cycle with radius $\sqrt{r_{k}}$.

2.2. Coupling Dynamics. Associated to an $n$ vertex connected and undirected network, denote by $A$ the weighted adjacency matrix with positive entries $a_{k, j} \geqslant 0$. Let $L$ be the corresponding Laplacian matrix $(L:=D-A$, where $D$ is the diagonal matrix with $\left.d_{k, k}:=\sum_{j=1}^{n} a_{k, j}\right)$. The coupling dynamics is given by the gradient of the positive semidefinite function

$$
\begin{aligned}
\mathrm{V}(\phi, r) & :=\frac{1}{2}\left\langle r \mid L_{\cos } r\right\rangle \\
& =\frac{1}{2} \sum_{k=1}^{n} r_{k} \sum_{j=1}^{n} l_{k, j} r_{j} \cos \left(\phi_{k}-\phi_{j}\right) \geqslant 0
\end{aligned}
$$

with $\phi=\left(\phi_{1}, \ldots, \phi_{n}\right)$ and $r=\left(r_{1}, \ldots, r_{n}\right)$ and where the matrix $L_{\text {cos }}$ has entries $l_{k, j} \cos \left(\phi_{k}-\phi_{j}\right)$. This matrix is positive semidefinite since all its eigenvalues are positive (i.e. nonnegative). This is a direct application of Gershgorin's circle theorem [11]: for any eigenvalue $\zeta_{\text {cos }}$ of $L_{\text {cos }}$, there exists $k$ such that

$$
\left|\zeta_{\cos }-l_{k, k}\right| \leqslant \sum_{j \neq k}^{n}\left|l_{k, j} \cos \left(\phi_{k}-\phi_{j}\right)\right|
$$

and so $\left|\zeta_{\text {cos }}-l_{k, k}\right| \leqslant \sum_{j \neq k}^{n}\left|l_{k, j} \cos \left(\phi_{k}-\phi_{j}\right)\right| \leqslant \sum_{j \neq k}^{n}\left|l_{k, j}\right|=l_{k, k}$. In particular, the coupling dynamics is

$$
\begin{aligned}
& \mathrm{c}_{k} \frac{\partial \mathrm{V}}{\partial \phi_{k}}(\phi, r)=-\mathrm{c}_{k} \sum_{j=1}^{n} l_{k, j} r_{k} r_{j} \sin \left(\phi_{k}-\phi_{j}\right), \quad k=1, \ldots, n, \\
& \mathrm{c}_{k} \frac{\partial \mathrm{V}}{\partial r_{k}}(\phi, r)=\mathrm{c}_{k} \sum_{j=1}^{n} l_{k, j} r_{j} \cos \left(\phi_{k}-\phi_{j}\right), \quad k=1, \ldots, n,
\end{aligned}
$$

where $c_{k}>0$ are coupling strengths.

2.3. Adaptive Mechanisms. In this section, we now allow the fixed and constant parameters $\mathrm{w}_{k}$ and $\mathrm{r}_{k}$ to

(I) become time dependent, that is, $\Omega_{k}=\left\{\mathrm{w}_{k}, \mathrm{r}_{k}\right\} \leadsto$ $\left(\omega_{k}(t), \rho_{k}(t)\right)=\Omega_{k}(t)$, for $k=1, \ldots, n$,

(II) and each of them have their own dynamics, depending solely on the state variables $\phi$ and $r$, that is,

$$
\mathrm{A}_{k}(\phi, r)=\left(\mathrm{A}_{k}^{\mathrm{w}}(\phi, r), \mathrm{A}_{k}^{\mathrm{r}}(\phi, r)\right) \quad \text { for } k=1, \ldots, n \text {. }
$$

Among the numerous variants for changing the values of the parameter, we focus on those presented in $[7,8,12]$, that is,

$$
\begin{aligned}
& \mathrm{A}_{k}^{\mathrm{w}}(\phi, r)=\mathrm{s}_{k} \sum_{j=1}^{n} l_{k, j} r_{k} r_{j} \sin \left(\phi_{k}-\phi_{j}\right), \quad k=1, \ldots, n, \\
& \mathrm{~A}_{k}^{\mathrm{r}}(\phi, r)=-\mathrm{s}_{k} \sum_{j=1}^{n} l_{k, j} r_{j}^{2}, \quad k=1, \ldots, n,
\end{aligned}
$$

where $l_{k, j}$ are the entries of $L$ and $s_{k}$ are "susceptibility constants": the larger $s_{k}$ is, the stronger the influence on $\omega_{k}$ and $\rho_{k}$ is. Conversely, oscillators with small $s_{k}$ are reluctant to modify their frequency and their limit cycle radius.

\section{Network's Dynamical System with Delay}

We now discuss the resulting dynamics in the presence of a time delay $t \geqslant 0$ affecting both the coupling dynamics and the adaptive mechanisms. We hence consider

$$
\begin{aligned}
\dot{\phi}_{k}(t)= & \omega_{k}(t)+\mathrm{c}_{k} \sum_{j=1}^{n} l_{k, j} r_{k}(t-\mathrm{t}) r_{j}(t-\mathrm{t}) \\
& \times \sin \left(\phi_{k}(t-\mathrm{t})-\phi_{j}(t-\mathrm{t})\right), \\
\dot{r}_{k}(t)= & -\left(r_{k}(t)^{2}-\rho_{k}(t)\right) r_{k}(t) \\
& -\mathrm{c}_{k} \sum_{j=1}^{n} l_{k, j} r_{j}(t-\mathrm{t}) \cos \left(\phi_{k}(t-\mathrm{t})-\phi_{j}(t-\mathrm{t})\right), \\
\dot{\omega}_{k}(t)= & \mathrm{s}_{k} \sum_{j=1}^{n} l_{k, j} r_{k}(t-\mathrm{t}) r_{j}(t-\mathrm{t}) \sin \left(\phi_{k}(t-\mathrm{t})-\phi_{j}(t-\mathrm{t})\right), \\
\dot{\rho}_{k}(t)= & -s_{k} \sum_{j=1}^{n} l_{k, j} r_{j}(t-\mathrm{t})^{2},
\end{aligned}
$$

for $k=1, \ldots, n$. For $(14)$, we have the following.

Two Constants of Motion. The functions

$$
\mathrm{J}(\omega)=\sum_{j=1}^{n} \frac{\omega_{j}}{\mathrm{~s}_{j}}, \quad \mathrm{~K}(\rho)=\sum_{j=1}^{n} \frac{\rho_{j}}{\mathrm{~s}_{j}}
$$

are constants of motion: in other words, if $\omega(t)=\left(\omega_{1}(t), \ldots\right.$, $\left.\omega_{n}(t)\right)$ and $\rho(t)=\left(\rho_{1}(t), \ldots, \rho_{n}(t)\right)$ are orbits of $(14)$, then

$$
\begin{aligned}
\frac{\mathrm{d}[\mathrm{J}(\omega(t))]}{\mathrm{d} t} & =\langle\nabla \mathrm{J}(\omega(t)) \mid \dot{\omega}(t)\rangle \\
& =\frac{\mathrm{d}[\mathrm{K}(\rho(t))]}{\mathrm{d} t}=\langle\nabla \mathrm{K}(\rho(t)) \mid \dot{\rho}(t)\rangle=0 .
\end{aligned}
$$


Existence of a Consensual Oscillatory State. We can explicitly exhibit a consensual oscillatory state. Indeed, for given $\omega_{c}$ and $\rho_{c}>0$

$$
\left(\phi_{k}(t), r_{k}(t), \omega_{k}(t), \rho_{k}(t)\right):=\left(\omega_{c} t, \sqrt{\rho_{c}}, \omega_{c}, \rho_{c}\right)
$$

for all $t \in[-\mathrm{t}, 0] \cup \mathbb{R}_{\geqslant 0}$ is a consensual orbit of (14).

Observe that in absence of the radial component $r_{k}$ and without adaptation, (14) yields the famous Kuramoto model with delays $[13,14]$. In these contributions, the authors considered the coupling dynamics with delay of the form: $\sum_{j=1}^{n} l_{k, j} \sin \left(\phi_{k}(t)-\phi_{j}(t-\mathrm{t})\right)$, that is, delays concern the "exterior" variables $\phi_{j}$ and not the "local" variable $\phi_{k}$. This can be done here for the coupling dynamics but not for the adaptive mechanism on $\omega_{k}$, if we require $\mathrm{J}$ to be a constant of motion J.

In the absence of time delay (i.e., $\mathrm{t}=0$ ) and under appropriate conditions (c.f. [12] for details), the adaptive mechanisms tune the value of frequencies $\omega_{k}$ and the radii $\rho_{k}$ of the attractors so that the global dynamical system is driven into a consensual oscillatory state. In other words, we have the following limit:

$$
\begin{aligned}
\lim _{t \rightarrow \infty} & \left\|\left(\phi_{k}(t), r_{k}(t), \omega_{k}(t), \rho_{k}(t)\right)-\left(\omega_{c} t, \sqrt{\rho_{c}}, \omega_{c}, \rho_{c}\right)\right\| \\
& =0 \quad \forall k,
\end{aligned}
$$

with constants $\omega_{c}$ and $\rho_{c}$ and where $\|\cdot\|$ is the Euclidean norm. The consensual state is permanent (i.e., even if interactions are switched off, all local dynamics still oscillate with the same frequency and same amplitude). Let us now discuss the conditions for which Limit (18) holds when the global dynamics is affected by a time delay (i.e., $t>0$ ).

Convergence towards a Consensual Oscillatory State. The Limit (18) raises two issues: (1) the existence itself and (2) the limit values $\omega_{c}$ and $\rho_{c}$. For expository reasons, we first discuss the limit values and then the convergence conditions.

Limit Values. Thanks to the constant of motions in (15), we have

$$
\mathrm{J}(\omega(0))=\mathrm{J}(\omega(t)) \quad \forall t, \quad \mathrm{~K}(\rho(0))=\mathrm{K}(\rho(t)) \quad \forall t,
$$

with $\omega(t)=\left(\omega_{1}(t), \ldots, \omega_{n}(t)\right)$ and $\rho(t)=\left(\rho_{1}(t), \ldots, \rho_{n}(t)\right)$ orbits of (14) with given initial conditions. Supposing that Limit (18) holds, we hence have

$$
\begin{aligned}
& \mathrm{J}(\omega(0))=\lim _{t \rightarrow \infty} \mathrm{J}(\omega(t))=\omega_{c} \sum_{j=1}^{n} \frac{1}{\mathrm{~s}_{j}}, \\
& \mathrm{~K}(\rho(0))=\lim _{t \rightarrow \infty} \mathrm{K}(\rho(t))=\rho_{c} \sum_{j=1}^{n} \frac{1}{\mathrm{~s}_{j}},
\end{aligned}
$$

and so the asymptotic values are analytically expressed as

$$
\omega_{c}=\frac{\sum_{j=1}^{n}\left(\omega_{j}(0) / s_{j}\right)}{\sum_{j=1}^{n}\left(1 / s_{j}\right)}, \quad \rho_{c}=\frac{\sum_{j=1}^{n}\left(\rho_{j}(0) / s_{j}\right)}{\sum_{j=1}^{n}\left(1 / s_{j}\right)} .
$$

It is important to emphasize that the consensual values $\omega_{c}$ and $\rho_{c}$ do not depend on the network topology (i.e., not on $L$ ), nor on the initial conditions of the state variables (i.e., not on $\left(\phi_{k}(0), r_{k}(0)\right)$ nor on the time delay (i.e., not on $\mathrm{t}$ ).

Convergence Conditions. To this aim we study the first-order approximation of (14) in the vicinity of Solution (17) and assume that linear stability analysis is sufficient to infer convergence conditions for the nonlinear system. Accordingly, we study the asymptotic behavior of the small perturbations $\epsilon_{\phi_{k}}(t), \epsilon_{r_{k}}(t), \epsilon_{\omega_{k}}(t)$, and $\epsilon_{\rho_{k}}(t)$ and write

$$
\begin{aligned}
& \left(\phi_{k}(t), r_{k}(t), \omega_{k}(t), \rho_{k}(t)\right) \\
& \quad=\left(\omega_{c} t+\epsilon_{\phi_{k}}(t), \sqrt{\rho_{c}}+\epsilon_{r_{k}}(t), \omega_{c}+\epsilon_{\omega_{k}}(t), \rho_{c}+\epsilon_{\rho_{k}}(t)\right) .
\end{aligned}
$$

Taking into account the constant of motions, we impose that

$$
\sum_{j=1}^{n} \frac{\epsilon_{\omega_{j}}(0)}{s_{j}}=0, \quad \sum_{j=1}^{n} \frac{\epsilon_{\rho_{j}}(0)}{s_{j}}=0 .
$$

First Order Approximation. Rearranging the variables (i.e., the first $n$ are the $\phi_{k}$, the second $n$ are the $r_{k}$, the third $n$ are the $\omega_{k}$, and finally the last $n$ are the $\rho_{k}$ ), the first-order approximation of (14) is

$$
\begin{aligned}
\left(\begin{array}{c}
\dot{\epsilon}_{\phi} \\
\dot{\epsilon}_{r} \\
\dot{\epsilon}_{\omega} \\
\dot{\epsilon}_{\rho}
\end{array}\right)= & \left(\begin{array}{cccc}
\mathbf{0} & \mathbf{0} & I d & \mathbf{0} \\
\mathbf{0} & -2 \rho_{c} I d & \mathbf{0} & \sqrt{\rho_{c}} I d \\
\mathbf{0} & \mathbf{0} & \mathbf{0} & \mathbf{0} \\
\mathbf{0} & \mathbf{0} & \mathbf{0} & \mathbf{0}
\end{array}\right)\left(\begin{array}{l}
\epsilon_{\phi} \\
\epsilon_{r} \\
\epsilon_{\omega} \\
\epsilon_{\rho}
\end{array}\right) \\
& +\left(\begin{array}{cccc}
-\rho_{c}[\mathrm{c}] L & \mathbf{0} & \mathbf{0} & \mathbf{0} \\
\mathbf{0} & -[\mathrm{c}] L & \mathbf{0} & \mathbf{0} \\
-\rho_{c}[\mathrm{~s}] L & \mathbf{0} & \mathbf{0} & \mathbf{0} \\
\mathbf{0} & -2 \sqrt{\rho_{c}}[\mathrm{~s}] L & \mathbf{0} & \mathbf{0}
\end{array}\right)\left(\begin{array}{c}
\check{\epsilon}_{\phi} \\
\check{\epsilon}_{r} \\
\check{\epsilon}_{\omega} \\
\check{\epsilon}_{\rho}
\end{array}\right),
\end{aligned}
$$

with the $n \times n$ identity matrix $I d$, diagonal matrices [c] and [s] with, respectively, the coupling strengths and the susceptibility constants as entries and $\epsilon_{\phi}:=\left(\epsilon_{\phi_{1}}, \ldots, \epsilon_{\phi_{n}}\right), \epsilon_{r}:=\left(\epsilon_{r_{1}}\right.$, $\left.\ldots, \epsilon_{r_{n}}\right), \epsilon_{\omega}:=\left(\epsilon_{\omega_{1}}, \ldots, \epsilon_{\omega_{n}}\right)$ and $\epsilon_{\rho}:=\left(\epsilon_{\rho_{1}}, \ldots, \epsilon_{\rho_{n}}\right)$, and where the delayed perturbations are $\check{\epsilon}_{\phi}=\epsilon_{\phi}(t-\mathrm{t}), \check{\epsilon}_{r}=\epsilon_{r}(t-\mathrm{t})$, $\check{\epsilon}_{\omega}=\epsilon_{\omega}(t-\mathrm{t}) \check{\epsilon}_{\rho}=\epsilon_{\rho}(t-\mathrm{t})$.

Diagonalization. Suppose now that $[\mathrm{s}]=\mathrm{q}[\mathrm{c}]$ for some positive constant $\mathrm{q}$ and let $\mathrm{O}$ denote an orthogonal matrix (i.e., $\left.O^{\top} O=O O^{\top}=I d\right)$ with real entries such that $O^{\top}[\mathrm{c}]^{1 / 2} L$ $[c]^{1 / 2} \mathrm{O}=[\zeta]$, with $[\zeta]$ being a diagonal matrix with the eigenvalues of the symmetric matrix $[c]^{1 / 2} L[c]^{1 / 2}$ on its diagonal. The signs of these coincide with those of the eigenvalues of $L$ : they are all strictly positive except for one, that is, zero. Hence, without loss of generality, one takes $\zeta_{1}=0$ and $\zeta_{k}>0$ for $k=2, \ldots, n$.

Changing the basis of System (24) with a $4 \times 4$ bloc matrix (each bloc of size $n \times n$ ) with $O^{\top}[\mathrm{c}]^{-1 / 2}$ on its diagonal, we 
can decompose the original system into $2 n$ 2-dimensional systems of the form

$$
\begin{aligned}
& \left(\begin{array}{c}
\dot{\varepsilon}_{\phi_{k}} \\
\dot{\varepsilon}_{\omega_{k}}
\end{array}\right)=\left(\begin{array}{ll}
0 & 1 \\
0 & 0
\end{array}\right)\left(\begin{array}{c}
\varepsilon_{\phi_{k}} \\
\varepsilon_{\omega_{k}}
\end{array}\right)+\left(\begin{array}{cc}
-\rho_{c} \zeta_{k} & 0 \\
-\rho_{c} \mathrm{q} \zeta_{k} & 0
\end{array}\right)\left(\begin{array}{c}
\check{\varepsilon}_{\phi_{k}} \\
\check{\varepsilon}_{\omega_{k}}
\end{array}\right), \\
& \left(\begin{array}{c}
\dot{\varepsilon}_{r_{k}} \\
\dot{\varepsilon}_{\rho_{k}}
\end{array}\right)=\left(\begin{array}{cc}
-2 \rho_{c} & \sqrt{\rho_{c}} \\
0 & 0
\end{array}\right)\left(\begin{array}{c}
\varepsilon_{r_{k}} \\
\varepsilon_{\rho_{k}}
\end{array}\right)+\left(\begin{array}{cc}
-\zeta_{k} & 0 \\
-2 \sqrt{\rho_{c}} \mathrm{q} \zeta_{k} & 0
\end{array}\right)\left(\begin{array}{c}
\check{\varepsilon}_{r_{k}} \\
\check{\varepsilon}_{\rho_{k}}
\end{array}\right),
\end{aligned}
$$

with $\varepsilon_{\phi}:=O^{\top}[\mathrm{c}]^{-1 / 2} \epsilon_{\phi}$ (resp., for $\epsilon_{r}, \epsilon_{\omega}, \epsilon_{\rho}$ ) and $\check{\varepsilon}_{\phi}:=$ $O^{\top}[\mathrm{c}]^{-1 / 2} \check{\epsilon}_{\phi}$ (resp. for $\check{\epsilon}_{r}, \check{\epsilon}_{\omega}, \check{\epsilon}_{\rho}$ ) for delayed perturbations obtained after the change of basis.

The case $k=1$ is worked out in the appendix. For $k \neq 1$, let us focus on the 2-dimensional systems and we rewrite (25a)-(25b) as linear second-order time delayed differential equations

$$
\begin{gathered}
\ddot{\varepsilon}_{\phi_{k}}(t)+\rho_{c} \zeta_{k} \dot{\varepsilon}_{\phi_{k}}(t-\mathrm{t})+\rho_{c} \mathrm{q} \zeta_{k} \varepsilon_{\phi_{k}}(t-\mathrm{t})=0, \\
\ddot{\varepsilon}_{r_{k}}(t)+2 \rho_{c} \dot{\varepsilon}_{r_{k}}(t)+\zeta_{k} \dot{\varepsilon}_{r_{k}}(t-\mathrm{t})+2 \rho_{c} \mathrm{q} \zeta_{k} \varepsilon_{r_{k}}(t-\mathrm{t})=0 .
\end{gathered}
$$

The convergence towards a consensual state is hence determined by the asymptotic stability of the zero solution of (26). Stability follows if, and only if, all roots of the corresponding characteristic equations have strictly negative real parts (c.f. [15] for details). For (25a), one can apply Theorem 3.3 in [16] which states that in this case the zero solution is asymptotically stable if and only if

(I)

$$
\mathrm{t} \rho_{c} \zeta_{k}<\frac{\pi}{2}
$$

(II)

$$
\begin{gathered}
\frac{\mathrm{t}^{2} \rho_{c} \mathrm{q} \zeta_{k}}{z^{2}}<\cos (z), \text { where } z \text { is the unique solution in } \\
] 0, \frac{\pi}{2}\left[\text { of } \sin (z)=\frac{\mathrm{t} \rho_{c} \zeta_{k}}{z},\right.
\end{gathered}
$$

for $k=2, \ldots, n$. We emphasize that the consensual value $\rho_{c}$ influences the condition for convergence whereas it is not the case for $\omega_{c}$. This leads to the idea that shaping the attractor is more delicate than tuning the angular velocity. This has been observed in [17].

Adaptation only on $\omega_{k}$. If there is no adaptation on the radii (i.e., $\rho_{k}(t):=r$ for all $k$ and $t$ ), then (25b) reduced to

$$
\dot{\varepsilon}_{r_{k}}(t)=-2 r \varepsilon_{r_{k}}(t)-\zeta_{k} \varepsilon_{r_{k}}(t-\mathrm{t})
$$

for which the zero solution is asymptotically stable provided (c.f. [18] for details)

$$
4 \mathrm{r}^{2}<\zeta_{k}^{2}, \quad \mathrm{t}<\check{\mathrm{t}} \text { with } \check{\mathrm{t}}=\frac{\cot ^{-1}\left(-2 \mathrm{r} / \sqrt{\zeta_{k}^{2}-4 \mathrm{r}^{2}}\right)}{\sqrt{\zeta_{k}^{2}-4 \mathrm{r}^{2}}} .
$$

The zero solution is unstable when $\mathrm{t}>\check{\mathrm{t}}$. Note that stability is guaranteed for any $\mathrm{t}$ when $\zeta_{k}^{2}<4 \mathrm{r}^{2}$ or $\zeta_{k}^{2}=4 \mathrm{r}^{2} \neq 0$.

No Time Delay in the Coupling Dynamics. If there is no time delay in the coupling dynamics (i.e., the coupling dynamics is defined as in (11) with no delay), then (26) becomes, for $k=2, \ldots, n$,

$$
\begin{gathered}
\ddot{\varepsilon}_{\phi_{k}}(t)+\rho_{c} \zeta_{k} \dot{\varepsilon}_{\phi_{k}}(t)+\rho_{c} \mathrm{q} \zeta_{k} \varepsilon_{\phi_{k}}(t-\mathrm{t})=0, \\
\ddot{\varepsilon}_{r_{k}}(t)+\left(2 \rho_{c}+\zeta_{k}\right) \dot{\varepsilon}_{r_{k}}(t)+2 \rho_{c} \mathrm{q} \zeta_{k} \varepsilon_{r_{k}}(t-\mathrm{t})=0 .
\end{gathered}
$$

Invoking Theorem 3.5 in [16], the zero solution for (30a) and (30b) is asymptotically stable if and only if.

For (30a)

$$
\begin{aligned}
& \mathrm{t}^{2} \rho_{c} \mathrm{q} \zeta_{k}+\left(z^{2}+\mathrm{t}^{2}\left(\rho_{c} \zeta_{k}\right)^{2}\right) \cos (z)>0 \text {, where } z \text { is the } \\
& \text { unique solution in }] 0, \frac{\pi}{2}\left[\text { of } z \sin (z)=\mathrm{t} \rho_{c} \zeta_{k} \cos (z)\right. \text {, }
\end{aligned}
$$

for $(30 b)$

$$
\begin{gathered}
-2 \mathrm{t}^{2} \rho_{c} \mathrm{q} \zeta_{k}+\left(z^{2}+\mathrm{t}^{2}\left(2 \rho_{c}+\zeta_{k}\right)^{2}\right) \cos (z)>0 \text {, where } z \text { is } \\
\text { the unique solution in }] 0, \frac{\pi}{2}[\text { of } z \sin (z) \\
=\mathrm{t}\left(2 \rho_{c}+\zeta_{k}\right) \cos (z),
\end{gathered}
$$

for $k=2, \ldots, n$.

Summary. For a network (with arbitrary topology but with symmetric, positive entries adjacency matrix) of Hopf oscillators (as defined in Section 2.1 by (8)) interacting through time delayed Kuramoto type coupling (as defined in Section 2.2 by (11)) and with time-delay adaptive mechanisms (as defined in Section 2.3 by (13)) on the frequencies and amplitudes of the local systems-in other words, for (14), we have the following.

(I) two constants of motions (c.f. (15)),

(II) the existence of a consensual oscillatory state (c.f. (17)),

(III) the consensual oscillatory state is linearly (i.e., locally) stable if all the roots of characteristic equations corresponding to (26) have strictly negative real parts,

(IV) if there is no adaptation on the radii, the consensual oscillatory state is linearly (i.e., locally) stable if (27a), (27b), and (29) hold,

(V) if there is no delay in the coupling dynamics, the consensual oscillatory state is linearly (i.e., locally) stable if (31), and (32) hold.

3.1. Miscellaneous Remark: Delayed Stabilization Mechanism. In this section we discuss the particular case arising when the delay is introduced only in the stabilization mechanism (i.e., 
dissipative part) of the local dynamics. The dynamical system is

$$
\begin{aligned}
\dot{\phi}_{k}(t)= & \omega_{k}(t)+c_{k} \sum_{j=1}^{n} l_{k, j} r_{k}(t) r_{j}(t) \sin \left(\phi_{k}(t)-\phi_{j}(t)\right), \\
k=1, \ldots, n, & \\
\dot{r}_{k}(t)= & -r_{k}\left((t-\mathrm{t})^{2}-\rho_{k}(t)\right) r_{k}(t) \quad k=1, \ldots, n, \\
& -c_{k} \sum_{j=1}^{n} l_{k, j} r_{j}(t) \cos \left(\phi_{k}(t)-\phi_{j}(t)\right), \quad k=1, \ldots, n, \\
\dot{\omega}_{k}(t)= & s_{k} \sum_{j=1}^{n} l_{k, j} r_{k}(t) r_{j}(t) \sin \left(\phi_{k}(t)-\phi_{j}(t)\right), \quad k=1, \ldots, n . \\
\dot{\rho}_{k}(t)= & -s_{k} \sum_{j=1}^{n} l_{k, j} r_{j}(t)^{2}, \quad k=1,
\end{aligned}
$$

Equations (33) still admit the existence of a consensual oscillatory state and two constants of motion as in (15) and in (17), respectively. Linear stability analysis of the consensual state reduces to the study of

$$
\begin{gathered}
\ddot{\varepsilon}_{\phi_{k}}(t)+\rho_{c} \zeta_{k} \dot{\varepsilon}_{\phi_{k}}(t)+\rho_{c} \mathrm{q} \zeta_{k} \varepsilon_{\phi_{k}}(t)=0, \\
\ddot{\varepsilon}_{r_{k}}(t)+2 \rho_{c} \dot{\varepsilon}_{r_{k}}(t-\mathrm{t})+2 \rho_{c} \mathrm{q} \zeta_{k} \varepsilon_{r_{k}}(t)=0,
\end{gathered}
$$

for $k=2, \ldots, n$. The zero solution for (34a) is asymptotically stable. For (34b), we apply Theorem 3.4 in [16] which states that in this case the zero solution is asymptotically stable if and only if

(I)

$$
\begin{aligned}
\exists z \in \mathbb{Z}_{\geq 0}:=\{0,1,2, \ldots\} & \text { such that } \\
(2 z-1) \pi+\left(\frac{\pi}{2}\right) & <\mathrm{t} \sqrt{2 \rho_{c} q \zeta_{k}} \\
& <2 z \pi+\left(\frac{\pi}{2}\right)
\end{aligned}
$$

(II)

$$
\begin{aligned}
0>-2 \mathrm{t} \rho_{c}>\max \{ & (2 z-1) \pi+\left(\frac{\pi}{2}\right) \\
& -\left(\frac{2 \mathrm{t}^{2} \rho_{c} \mathrm{q} \zeta_{k}}{(2 z-1) \pi+(\pi / 2)}\right), \\
& -\left(2 z \pi+\left(\frac{\pi}{2}\right)\right) \\
& \left.+\left(\frac{2 \mathrm{t}^{2} \rho_{c} \mathrm{q} \zeta_{k}}{(2 z \pi+(\pi / 2))}\right)\right\},
\end{aligned}
$$

for $k=2, \ldots, n$.

\section{Applications}

Conceptually, the problem of reliably distributing time and frequency among several spatial remote locations is a "leitmotiv" in applications ranging from basic metrology, navigation and position determination, signal processing, computer communications, energy distribution networks, swarms robotics, bioengineering, multiagents systems, life sciences, acoustics, and musical art to give but only a highly nonexhaustive list. Presently, a strong research impetus is devoted to complex interacting oscillating systems able to exhibit self-adaptive capabilities leading to a resilient consensual dynamic. Whatever the configurations under study, communication delays between the collection of interacting subparts of the global systems are physically unavoidable. Depending on the underlying time scales, delays do strongly affect the resulting dynamics. Our class of models explicitly study the influence of delays and in particular their destabilizing effects, that modify the instantaneous behavior. By an appropriate tuning of control parameters (e.g., susceptibility constants), our class of models offer, via a unique formalism, the possibility to continuously explore interacting configurations ranging from slave-master (i.e., system-environment relationship) to fully decentralized regimes.

Alternatively, we may view this problematic in the context of soft-controlled systems which presently receive a sustain attention [19]. Here, a swarm of agents is infiltrated by a lure agent (sometimes called a shill in economy). While the lure exhibits all the features of any ordinary agent, it can be externally controlled by an operator. As the interactions between the lure and any agent of the swarm remain unaffected (i.e., the lure remains incognito to ordinary agents), the external control of the lure can ultimately drive the whole population to a specific configuration. In our class of dynamics, a suitable choice of the susceptibility constant of a given local system (i.e., oscillator) may convert it into a shill. Indeed, in view of (21), the ultimate consensual values $\omega_{c}$ and $\rho_{c}$ are weighted averages. Such weighted averages can be made to be strongly dependent on a very insensitive shill-stubborn to any external influence (i.e., with a very low susceptibility constant).

In absence of time delays, convergence towards a consensual state is observed even for large heterogeneities (widely dispersed initial frequencies and radii and large discrepancies among the susceptibility constants). Hence, a shill agent can be easily introduced. However, our present study shows, that time delays restrict the conditions for convergence towards a consensual state. As a consequence, the implementation of a lure is more delicate matter (i.e., here, the dynamics is far more sensitive to the value taken by the susceptibility constants).

\section{Numerical Simulations}

Adaptation on $\omega_{k}$ and $\rho_{k}$. We consider four Hopf oscillators interacting on a network with topology as in Figure 1. We choose the coupling strengths and susceptibility constants as $\left\{c_{1}, \ldots, c_{4}\right\}=\{0.1,2,5,3\}$ and $s_{k}=0.8 c_{k}$ for $k=1, \ldots, 4$ (i.e., $q=0.8)$. The time delay is $\mathrm{t}=0.1$. The initial function (i.e., history) is $\left(\phi_{k}(t), r_{k}(t), \omega_{k}(t), \rho_{k}(t)\right)=(t, 1,1,1)$ for 


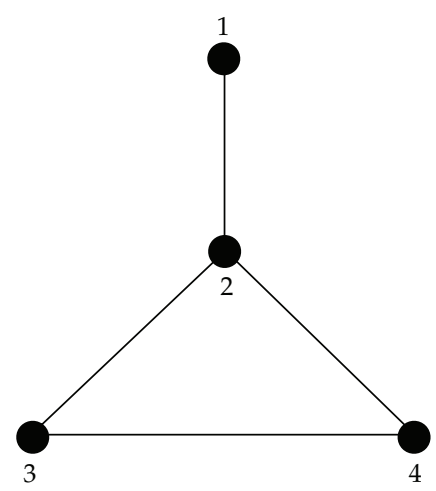

FIgURE 1: Network topology.

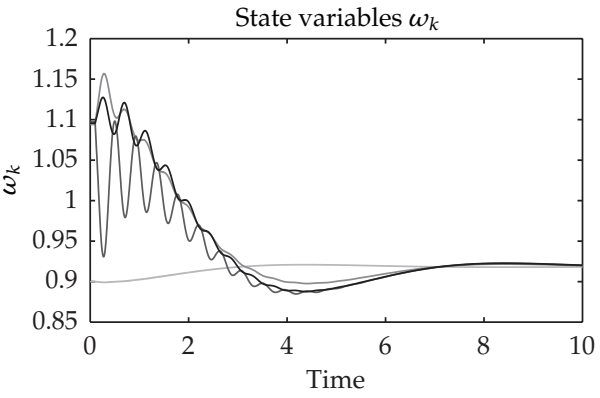

(a)

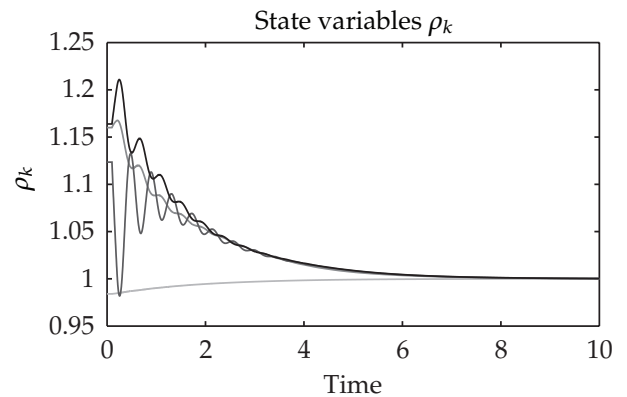

(b)

FIgure 2: Time evolution of $\omega_{k}$ (Figure 2(a)) and $\rho_{k}$ (Figure 2(b)) for four Hopf oscillators, interacting through the network in Figure 1. In both figures, all variables converge towards a constant and common consensual value.

$t \in\left[-\mathrm{t}, 0\left[\right.\right.$, having a jump at $t=0$ with values $\left(\phi_{k}(0), r_{k}(0)\right.$, $\left.\omega_{k}(0), \rho_{k}(0)\right)$ that are randomly uniformly drawn from $]-0.1$, $0.1[\times] 0.9,1.1[\times] 0.9,201.1[\times] 0.9,1.1[$ with the exception for $\omega_{1}(0)=0.9$. The $\rho_{k}(0)$ are rescaled such that the consensual value $\rho_{c}$ is one.

The resulting dynamics is shown in Figure 2. With the same initial conditions, we carry out another numerical simulation with here $t=0.12$. This violates Condition (27b) for $k=4$ and hence the network does not converge towards a consensual state. This is shown in Figure 3.

Note that in Figure 2(a), the $\omega_{k}(0)$ converge close to 0.9, that is, close to the initial value $\omega_{1}(0)$. This because the first oscillator's susceptibility is "small" and hence it is this local system that acts as a shill. It interacts with its neighbor in the

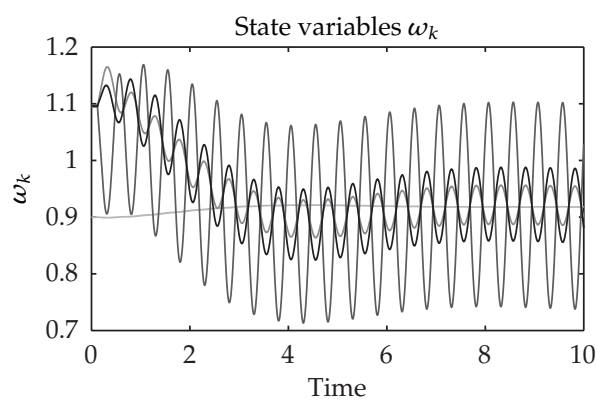

(a)

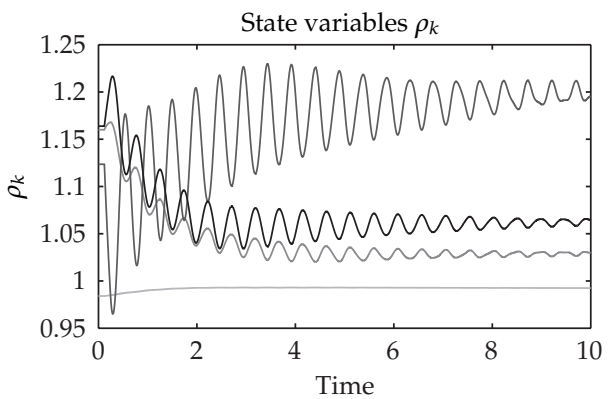

(b)

FIgURE 3: Time evolution of $\omega_{k}$ (Figure 3(a)) and $\rho_{k}$ (Figure 3(b)) for four Hopf oscillators, interacting through the network in Figure 1. In both figures, no common consensual value is reached.

same way as they act with it. It can control the behavior of the network and this without being connected to all other local systems.

In the extreme case, when $s_{k}=0$ for all $k$ (i.e., no adaptation), (14) describe the dynamics of coupled oscillators with different limit cycles and frequencies and delayed interactions. For small frequency and attractor's shape heterogeneities, the network is able to synchronize. In this case (i.e., without adaptive mechanisms for $\omega_{k}$ and $\rho_{k}$ ), several numerical simulations show that for delayed time $\mathrm{t}=0.1175$ synchronization is not systematically attained (i.e., it depends on the initial conditions)-whereas it is attained in the absence of the time delay (i.e., $\mathrm{t}=0$ ). On the other hand, when the adaptive mechanisms are switched on (i.e., $\mathrm{s}_{k}=$ $0.8 c_{k}>0$ for all $k$ ), a consensual state is reached-the linear stability criteria are still satisfied with $\mathrm{t}=0.1175$. Summarizing, here adaptation enhances the syntonization capability of the network.

Adaptation only on $\omega_{k}$. Two Hopf oscillators, both having the same radius for the attractor (i.e., $\rho_{k}=r=0.1$ for $k=$ $1,2)$, are coupled with coupling strengths and susceptibility constants as $\left\{c_{1}, c_{2}\right\}=\{1,14\}$ and $s_{k}=c_{k}$ for $k=1,2$ (i.e., $\mathrm{q}=1)$. The time delay is $\mathrm{t}=0.1057$. The initial function (i.e., history) is $\left(\phi_{k}(t), r_{k}(t), \omega_{k}(t)\right)=(t, \sqrt{0.1}, 1)$ for $t \in$ [-t, 0 [, having a jump at $t=0$ with values $\left(\phi_{k}(0), r_{k}(0)\right.$, $\left.\omega_{k}(0)\right)$ that are randomly uniformly drawn from ] -0.1 , $0.1[\times] \sqrt{0.1}-0.05, \sqrt{0.1}+0.05[\times] 0.9,1.1[$. Under this configuration, Conditions (27a)-(27b) are satisfied (hence the zero solution of (25a) is asymptotically stable) but 


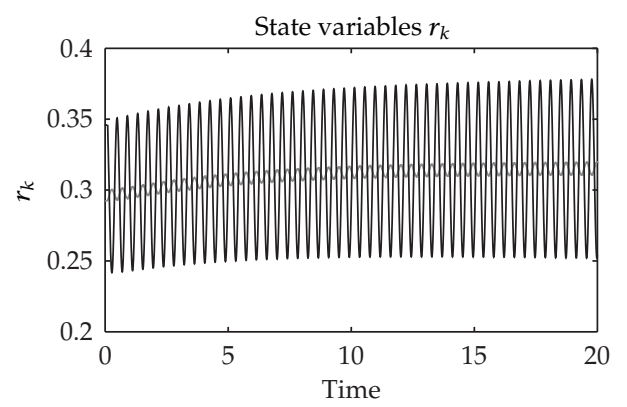

(a)

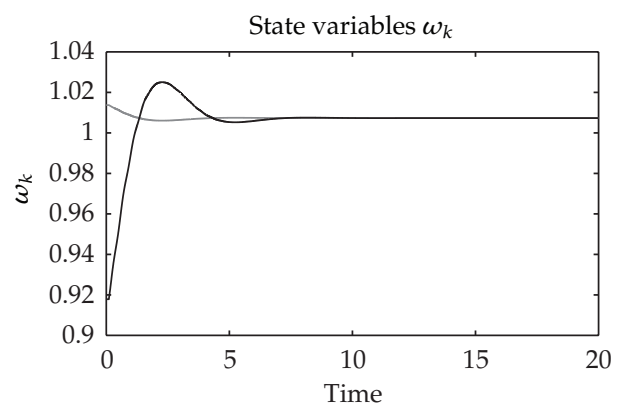

(b)

FIgURE 4: Time evolution of $r_{k}$ (Figure 4(a)) and $\omega_{k}$ (Figure 4(b)) for two Hopf oscillators. The two oscillators manage to adapt their frequencies while their radii do not converge towards their respective attractor.

not Condition (29) - the time delay is too large. Figure 4 displays the resulting dynamics. Observe that the radii do not converge towards their attractor. However, the oscillators still adapt their frequencies.

\section{Conclusion}

It is more the rule than the exception that parameter adaptation in dynamical systems can be achieved via delayed mechanisms. This therefore converts ordinary differential equations arising in absence of delay to functional differential equations. Stability issues become more difficult to discuss since, dealing with functional differential equations, an infinite number of degrees of freedom is introduced into the dynamics. For the class of oscillatory networks with parametric adaptation we here considered, we are able to observe how the time delay affects the adaptation mechanisms. While it is intuitively expected, that large delays are likely to destabilize the dynamics, we are here able to analytically quantify the underlying critical delays. The analytical linear stability discussion is made possible since, for our class of dynamics, stability issues can be reduced to the study of two linear second order functional differential equations for which suitable theorems can be found. Finally, we emphasize that numerical simulations show that adaptation may enhance the emergence of common dynamical pattern whereas in classical synchronization, time delays may be too large for synchronous motion to be attained.

\section{Appendix}

Assuming that all perturbations $\varepsilon_{\phi_{k}}, \varepsilon_{r_{k}}, \varepsilon_{\omega_{k}}, \varepsilon_{\rho_{k}}, \check{\varepsilon}_{\phi_{k}}, \check{\varepsilon}_{r_{k}}, \check{\varepsilon}_{\omega_{k}}$ and $\check{\varepsilon}_{\rho_{k}}$ for $k=2, \ldots, n$ converge to zero, let us now study the case for $k=1$. Here, $\zeta_{1}=0$ and so

$$
\begin{aligned}
& \dot{\varepsilon}_{\phi_{1}}=\varepsilon_{\omega_{1}}, \quad \dot{\varepsilon}_{\omega_{1}}=0, \\
& \dot{\varepsilon}_{r_{1}}=-2 \rho_{c} \varepsilon_{r_{1}}+\sqrt{\rho_{c}} \varepsilon_{\rho_{1}}, \quad \dot{\varepsilon}_{\rho_{1}}=0,
\end{aligned}
$$

and therefore $\varepsilon_{\omega_{1}}(t)=\varepsilon_{\omega_{1}}(0)$ and $\varepsilon_{\rho_{1}}(t)=\varepsilon_{\rho_{1}}(0)$ for all $t$. Both of these constants $\varepsilon_{\omega_{1}}(0)$ and $\varepsilon_{\rho_{1}}(0)$ are zero. This is because the first orthonormal base vector (i.e., the normalized eigenvector for the eigenvalue $\left.\zeta_{1}=0\right)$ is $\mathfrak{C}\left(1 / \sqrt{\mathbf{C}_{1}}, \ldots, 1 / \sqrt{\mathbf{C}_{n}}\right)$ (with $\left.\mathfrak{C}:=\left(\sum_{j=1}^{n}\left(1 / c_{j}\right)\right)^{-1 / 2}\right)$ and the first coordinate of $O^{\top}[\mathrm{c}]^{-1 / 2} \epsilon_{\omega}$ and $O^{\top}[\mathrm{c}]^{-1 / 2} \epsilon_{\rho}$ is, respectively,

$$
\begin{aligned}
& \varepsilon_{\omega_{1}}(0)=\mathfrak{c} \sum_{j=1}^{n} \frac{\epsilon_{\omega_{j}}(0)}{\mathrm{c}_{j}}=\mathfrak{c} \mathrm{q} \sum_{j=1}^{n} \frac{\epsilon_{\omega_{j}}(0)}{\mathrm{s}_{j}}, \\
& \varepsilon_{\rho_{1}}(0)=\mathfrak{c} \sum_{j=1}^{n} \frac{\epsilon_{\rho_{j}}(0)}{\mathrm{c}_{j}}=\mathfrak{s q} \sum_{j=1}^{n} \frac{\epsilon_{\rho_{j}}(0)}{\mathrm{s}_{j}},
\end{aligned}
$$

since we supposed that $s_{j}=\mathrm{qc}_{j}$ for all $j$. These two sums are zero according to (23). Therefore, $\dot{\varepsilon}_{r_{1}}=-2 \rho_{c} \varepsilon_{r_{1}}$ (i.e., $\left.\lim _{t \rightarrow \infty} \varepsilon_{r_{1}}(t)=0\right)$ and $\varepsilon_{\phi_{1}}(t)=\varepsilon_{\phi_{1}}(0)$ for all $t$. This allows one to conclude that all perturbations $\varepsilon_{r_{k}}, \varepsilon_{\rho_{k}}$, and $\varepsilon_{\omega_{k}}$ decay for all $k$. We now need to study how the perturbations on the phases evolve. Since $\epsilon_{\phi}=[c]^{1 / 2} O \epsilon_{\phi}$, then

$$
\begin{aligned}
\lim _{t \rightarrow \infty} \epsilon_{\phi_{k}}(t) & =\lim _{t \rightarrow \infty} \sqrt{\mathrm{c}_{k}} \sum_{j=1}^{n} o_{k, j} \varepsilon_{\phi_{j}}(t)=\sqrt{\mathrm{c}_{k}} o_{k, 1} \lim _{t \rightarrow \infty} \varepsilon_{\phi_{1}}(t) \\
& =\mathfrak{C} \varepsilon_{\phi_{1}}(0)=\mathfrak{c}^{2} \sum_{j=1}^{n} \frac{\epsilon_{\phi_{j}}(0)}{\mathrm{c}_{j}}=\frac{\sum_{j=1}^{n}\left(\epsilon_{\phi_{j}}(0) / \mathrm{c}_{j}\right)}{\sum_{j=1}^{n}\left(1 / \mathrm{c}_{j}\right)},
\end{aligned}
$$

since $\lim _{t \rightarrow \infty} \varepsilon_{\phi_{k}}(t)=0$ for $k=2, \ldots, n, o_{k, 1}=\mathfrak{c} 1 / \sqrt{\mathbf{c}_{k}}(k=$ $1, \ldots, n)$ and the first coordinate of the product $O^{\top}[c]^{-1 / 2} \epsilon_{\phi}$ is $\varepsilon_{\phi_{1}}(0)=\mathfrak{c} \sum_{j=1}^{n}\left(\epsilon_{\phi_{j}}(0) / c_{j}\right)$. Hence, all perturbations converge towards zero except those on the phase that all converge towards a constant (i.e., average phase perturbation). This corresponds to a phase shift.

\section{Acknowledgments}

M.-O. Hongler acknowledges partial support from the ESF (European Science Foundation) under the project entitled Exploring the Physics of Small Devices. J. Rodriguez acknowledges the support from the DFG-IRTG 1132 (Deutsche Forschungsgemeinschaft-International Research Training Group) under the project entitled Internationales Graduiertenkolleg-Stochastics and Real World Models. 


\section{References}

[1] A. A. Shabana, Theory of Vibration: An Introduction, Mechanical Engineering Series, Springer, New York, NY, USA, 1996.

[2] M. Lakshmanan and S. Rajasekar, Nonlinear Dynamics: Integrability, Chaos and Patterns, Advanced Texts in Physics, Springer, New York, NY, USA, 2003.

[3] J. Guckenheimer and P. Holmes, Nonlinear Oscillations, Dynamical Systems, and Bifurcations of Vector Fields, vol. 42 of Applied Mathematical Sciences, Springer, New York, NY, USA, 1990.

[4] J. Buchli, Ijspeert, and A. J. A. Simple, "Adaptive locomotion toysystem," in Proceedings of the 8th International Conference on the Simulation of Adaptive Behavior (SAB'04), 2004.

[5] L. Righetti, J. Buchli, and A. J. Ijspeert, "Dynamic Hebbian learning in adaptive frequency oscillators," Physica D, vol. 216, no. 2, pp. 269-281, 2006.

[6] R. Ronsse, N. Vitiello, T. Lenzi, J. van den Kieboom, M. C. Carrozza, and A. J. Ijspeert, "Human-robot synchrony: flexible assistance using adaptive oscillators," IEEE Transactions on Biomedical Engineering, vol. 58, no. 4, pp. 1001-1012, 2011.

[7] J. Rodriguez and M. O. Hongler, "Networks of mixed canonicaldissipative systems and dynamic hebbian learning," International Journal of Computational Intelligence Systems, vol. 2, no. 2, pp. 140-146, 2009.

[8] J. Rodriguez and M. O. Hongler, "Networks of limit cycle oscillators with parametric learning capability," in Recent Advances in Nonlinear Dynamics and Synchronization: Theory and Applications, K. Kyamakya, W. A. Halang, H. Unger, J. C. Chedjou, N. F. Rulkov, and Z. Li, Eds., pp. 17-48, Springer, Berlin, Germany, 2009.

[9] S. Steingrube, M. Timme, F. Wörgötter, and P. Manoonpong, "Self-organized adaptation of a simple neural circuit enables complex robot behaviour," Nature Physics, vol. 6, no. 3, pp. 224230, 2010 .

[10] A. A. Selivanov, J. Lehnert, T. Dahms, P. Hövel, A. L. Fradkov, and E. Schöll, "Adaptive synchronization in delay-coupled networks of Stuart-Landau oscillators," Physical Review E, vol. 85, no. 1, Article ID 016201, 8 pages, 2012.

[11] S. Gerschgorin, "Über die Abgrenzung der Eigenwerte einer Matrix," IzvestIya AkademII Nauk USSR, OtdelenIe MatematIcheskIh I estestvennIh Nauk, vol. 7, pp. 749-754, 1931.

[12] J. Rodriguez, Networks of self-adaptive dynamical systems [Ph.D. thesis], Ecole Polythechnique Fédérale de Lausanne, 2011.

[13] M. K. S. Yeung and S. H. Strogatz, "Time delay in the Kuramoto model of coupled oscillators," Physical Review Letters, vol. 82, no. 3, pp. 648-651, 1999.

[14] E. Montbrió, D. Pazó, and J. Schmidt, "Time delay in the Kuramoto model with bimodal frequency distribution," Physical Review E, vol. 74, no. 5, Article ID 056201, 5 pages, 2006.

[15] R. Bellman and K. L. Cooke, Delay Differential Equations with Applications in Populations Dynamics, Academic Press, New York, NY, USA, 1963.

[16] B. Cahlon and D. Schmidt, "Stability criteria for certain second order delay differential equations," Dynamics of Continuous, Discrete \& Impulsive Systems, vol. 10, no. 4, pp. 593-621, 2003.

[17] J. Rodriguez, M. O. Hongler, and P. Blanchard, "Self-adaptive attractor-shaping for oscillators networks," in Proceedings of the Joint INDS'11 \& ISTET'11-3rd International Workshop on nonlinear Dynamics and Synchronization and 6th International Symposium on Theoretical Electrical Engineering, 2011.
[18] Y. Kuang, Delay Differential Equations with Applications in Population Dynamics, vol. 191 of Mathematics in Science and Engineering, Academic Press, San Diego, Calif, USA, 1993.

[19] J. Han, M. Li, and L. Guo, "Soft control on collective behavior of a group of autonomous agents by a shill agent," Journal of Systems Science \& Complexity, vol. 19, no. 1, pp. 54-62, 2006. 


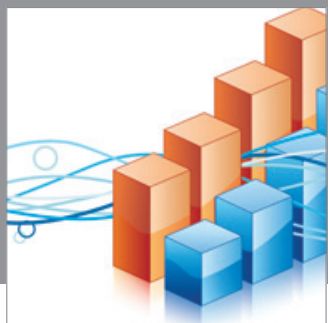

Advances in

Operations Research

mansans

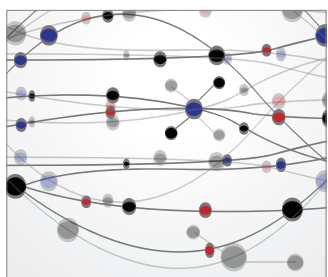

The Scientific World Journal
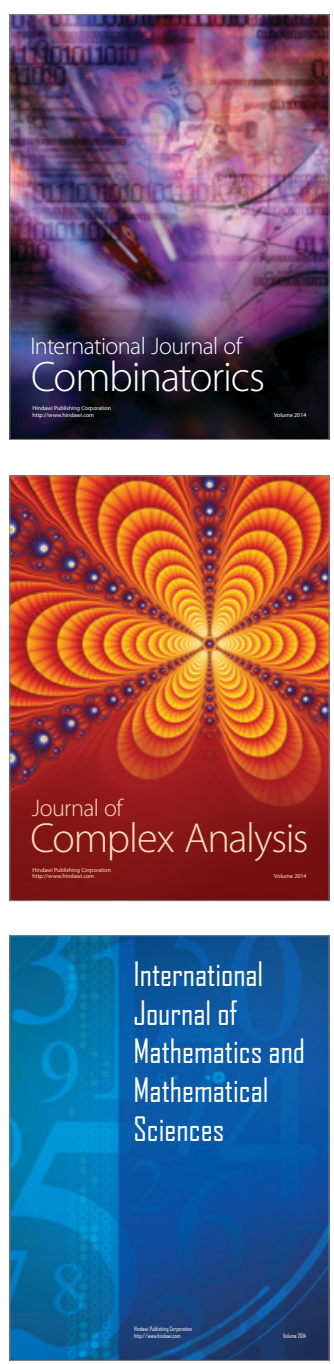
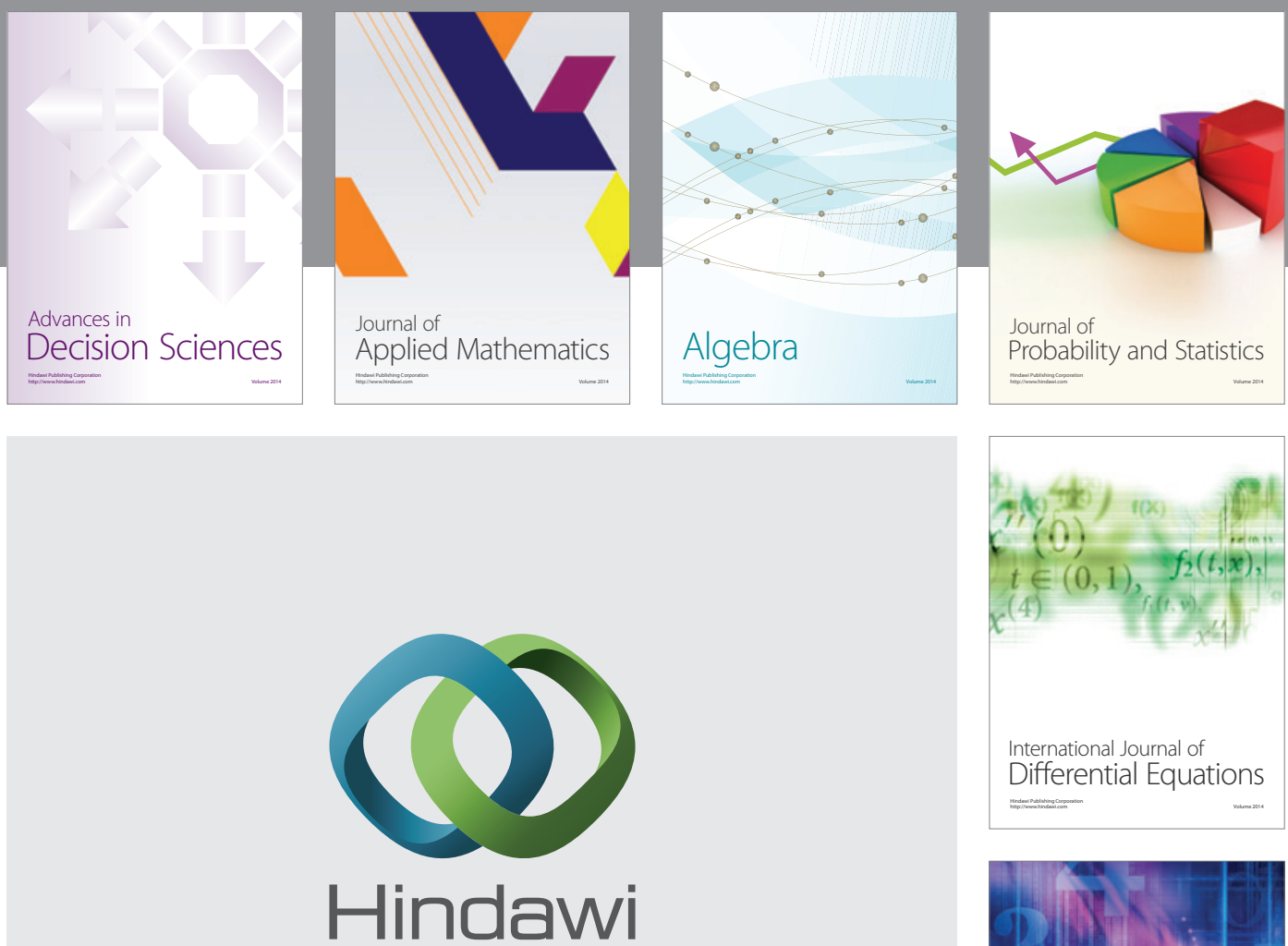

Submit your manuscripts at http://www.hindawi.com
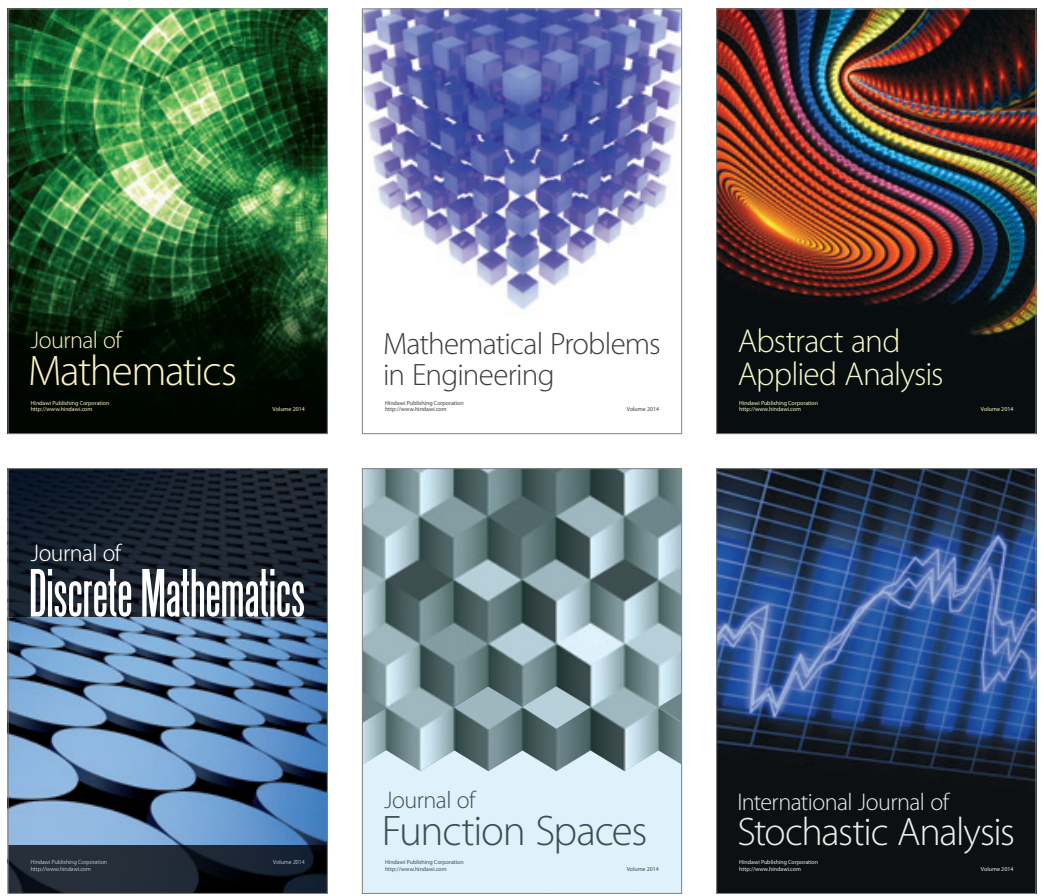

Journal of

Function Spaces

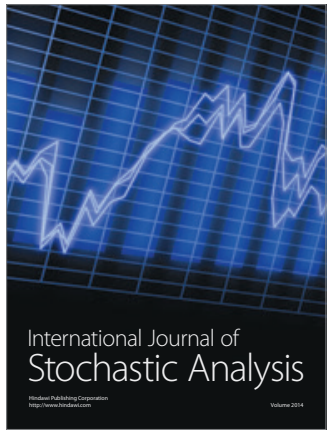

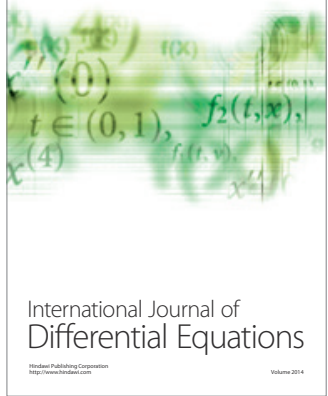
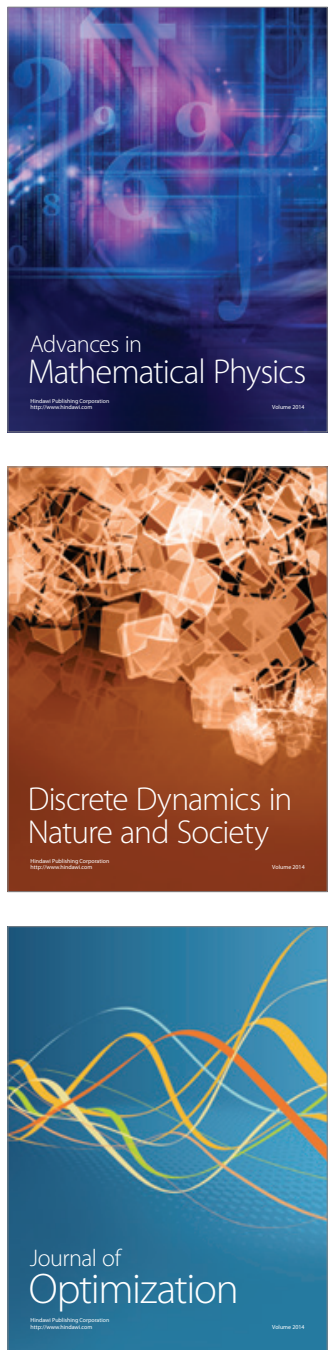